\title{
TEMA 2016: Vacuna contra virus del Papiloma Humano: Análisis de esquemas de dos dosificaciones
}
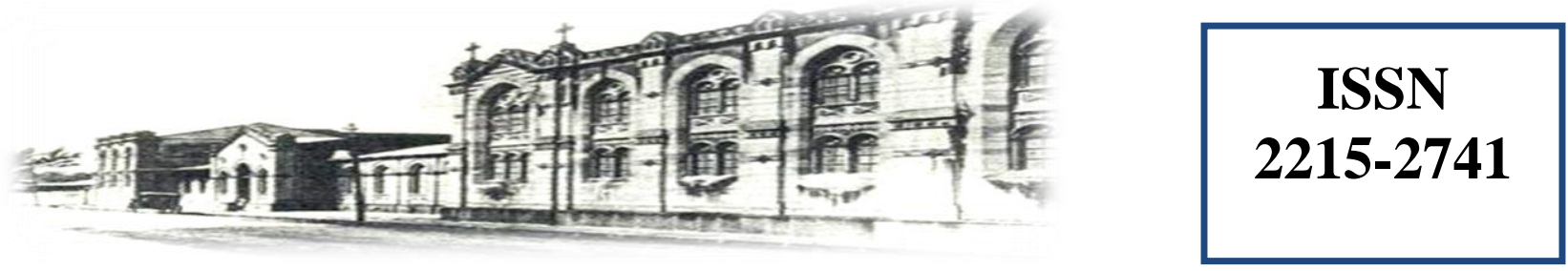

Hospital San Juan de Dios, San José, Costa Rica. Fundado en 1845

Recibido:

$10 / 02 / 2016$

Aceptado:

$15 / 06 / 2016$

\author{
Karen Acuña Rojas ${ }^{1}$ \\ Marco Vega Quesada ${ }^{2}$ \\ Nazaret Salazar Arias ${ }^{3}$ \\ Carlos Escalante Gómez ${ }^{4}$
}

\begin{abstract}
${ }^{1}$ Médico General. Universidad de Costa Rica. Correo electrónico: karen.1421@hotmail.com
${ }^{2}$ Médico General. Universidad de Costa Rica. Correo electrónico: marveque@gmail.com

${ }^{3}$ Médico General. Universidad de Costa Rica. Correo electrónico: nazaret.sa@hotmail.com

${ }^{4}$ Médico especialista en Ginecología y Obstetricia. Máster en Fisiología Humana. Académico de la Escuela de Medicina de la Universidad de Costa Rica: drescalante@gmail.com
\end{abstract}

\section{RESUMEN}

La infección por el virus del Papiloma Humano (VPH) es la enfermedad de transmisión sexual más frecuente alrededor del mundo, documentada tanto en mujeres como en hombres. Debido a su papel etiológico en el desarrollo de lesiones premalignas y malignas en diversos sitios anatómicos, se han desarrollado vacunas como herramientas en prevención primaria de estas neoplasias y principalmente en el cáncer de cérvix. Las vacunas aprobadas han demostrado ser altamente efectivas en evitar la transmisión del VPH y el desarrollo de enfermedades relacionadas con su infección, al ser utilizados en esquemas convencionales de tres dosis. Sin embargo, su alto costo y la falta de consistencia en sistemas de dosifica- ción son el principal obstáculo para el éxito. Es por esta razón, que en la actualidad se han realizado numerosos estudios basados en esquemas de dosificación reducidos y sencillos, en los que se compara su efectividad en relación los esquemas usuales, con el objetivo de facilitar la accesibilidad de la vacuna a la población más vulnerable.

\section{PALABRAS CLAVE}

Virus de Papiloma Humano. Vacunación. Proteínas semejantes al virus. Esquemas de dos dosis.

\section{ABSTRACT}


The infection by Human Papilloma Virus (HPV) is the most frequent sexual transmitted infection worldwide, documented in both genders. Due to its etiological roll on the development of premalignant and malignant lesions in different anatomic locations, several vaccines have been established as an important tool in primary care on the prevention of theses neoplasias, mainly on the battle against uterine cervix cancer. This vaccines had proven to be highly effective, preventing transmission and progression of other diseases related to HPV infection in usual treedose schedule. Although, the high cost of HPVvaccines and the difficulties to complete usual schedules are mayor obstacles to succeed. That is the reason why many recent studies have been focused on reduce and simplify schedules that can be compared on efficacy with usual tree-dose programs; with the purpose of increasing vaccine accessibility to the most vulnerable population.

\section{KEY WORDS}

Human papilloma virus. Vaccination. Virus like protein. Two doses schedules.

\section{INTRODUCCIÓN}

La infección por el VPH se ha reconocido como factor causal en la aparición de lesiones premalignas y malignas de cérvix, vagina, vulva, ano, pene y orofaringe $1,2,3,4,5$. El cáncer de cérvix se coloca en segundo lugar en cuanto a frecuencia a nivel mundial, con una mayor prevalencia en países subdesarrollados y siendo causa de una gran cantidad de muertes cada año ${ }^{2,6}$. Los serotipos 16 y 18 del VPH se observan hasta en el $70 \%$ de los casos de neoplasia a nivel de cérvix. Por tanto, la vacunación resulta una herramienta de gran importancia en la prevención de la infección, la cual a su vez ha demostrado gran eficacia en la prevención de estas neoplasias ${ }^{3}$.

Actualmente se presentan tres alternativas de vacunación aprobadas por la FDA: vacuna bivalente contra los serotipos 16 y 18 (Cervarix ${ }^{\circledR}$ de GlaxoSmithKline), vacuna tetravalente contra los serotipos 6, 11, 16 y 18 y la vacuna nonavalente que confiere protección tanto para los serotipo anteriores pero además incluye cinco serotipos oncogénicos; 31, 33, 45, 52 y 58 (Gardasil®4 y
Gardasil® 9, ambas de Merk\&Co). No obstante, esta última necesita de más estudios a largo plazo para valorar la eficacia e impacto, por lo que en esta revisión no será tomada en cuenta ${ }^{7,8}$.

Los esquemas de vacunación establecidos para las vacunas bivalente y tetravalente constan de tres dosis aplicadas en seis meses $(0,1$ y 6 meses y 0,2 y 6 meses; respectivamente). La vacuna se recomienda para mujeres entre los 9 y 14 años de edad ${ }^{8}$, idealmente antes del inicio de relaciones sexuales; sin embargo se benefician las mujeres entre los 9 a 26 años de edad y para los hombres entre los 9 y 26 años, en el caso de la vacuna tetravalente y la nonavalente ${ }^{1,7,9}$.

El desarrollo de planes de vacunación popular se han llevado a cabo en múltiples países desarrollados, sin embargo, estos no son comunes en países en subdesarrollo donde el alto costo de la vacuna representa un obstáculo significativo. Este hecho ha desalentado la extensión de la vacuna a los grupos que también se beneficiarían. Recientemente, la evidencia sugiere no inferioridad en cuanto a respuesta inmune en esquemas de solamente dos dosis comparado con los esquemas de 3 dosis; incluso indica que una dosis resulta en un aumento de anticuerpos mayor que al presentado después de la infección natural por el VPH ${ }^{10,11}$. La reducción del esquema de tres a dos dosis se ha llevado a cabo en países como Inglaterra y Canadá con resultados positivos ${ }^{10}$. En esta revisión se valora el esquema de dos dosis, el mecanismo y respuesta inmunológica ante las componentes vacunales, así como su costo efectividad.

\section{DISCUSIÓN}

Mecanismos inmunológicos involucrados en respuesta a infección natural por VPH

Se han reconocido cerca 130 serotipos distintos de VPH, que se pueden clasificar como precursores de lesiones benignos y precursores de lesiones malignas, este segundo grupo como fue mencionado con anterioridad representa cerca del $70 \%$ de las infecciones causadas por los seroti- 
pos 16 y 18, por esta razón se detallarán los mecanismos inmunológicos implicados en la infección por serotipos oncogénicos ${ }^{12}$.

El VPH es capaz de desarrollar una infección crónica evadiendo el sistema inmune, para comprender como es que el virus logra eludir la activación del sistema inmune del hospedero es necesario conocer el ciclo de vida del virus ${ }^{13}$. Este se inicia con la infección casi de manera exclusiva de los queratinocitos basales, células que de forma natural se diferencian y mueren sin necesidad de que el virus active mecanismos de citolisis o necrosis ${ }^{14}$. La invasión de los queratinocitos basales se consigue como consecuencia de su exposición durante la actividad sexual, producto de los microtraumas que se generan en el epitelio genital, principalmente en la zona de transformación ${ }^{13}$. Una vez que entran en contacto los receptores de las células basales con los productos de la cápside viral, se produce la internalización del virus hacia el citoplasma, sitio donde se fragmentan las proteínas virales, formando monómeros capaces de atravesar los poros nucleares de las células basales. Una vez en el núcleo se inician los procesos de replicación ${ }^{15}$.

Las células basales infectadas por el VPH inicialmente tienen niveles bajos de proliferación, se plantea que esta baja replicación permite la dispersión del virus por el estrato basal sin activar la respuesta inmune del hospedero. Posteriormente, se produce la expansión de las células infectadas hasta los estratos parabasal y espinoso, donde tiene lugar una etapa profilerativa más activa, así como la integración del genoma viral al genoma del hospedero ${ }^{15}$. Para el desarrollo de estos eventos, se han descrito modelos que señalan la participación de las proteínas virales E1, E2, E1-E4 y E6. Se conoce que la enzima E1 forma dímeros con la proteína E2 fosforilada, este dímero constituye el complejo de iniciación que se une a la región promotora del gen TATAbox, induciendo inhibición en la transcripción de los genes que codifican para las proteínas E6 y E7. Además, en la literatura se describe el papel de E2 en la activación de la proteína p53, detención del ciclo celular en G1 y apoptosis ${ }^{13}$.
En relación al complejo proteico E1-E4, se ha descrito su capacidad para generar alteraciones a nivel del citoesqueleto y en la función mitocondrial de la célula infectada. En conjunto estos procesos, con la supresión de proteínas oncogénicas y activación de señales apoptóticas, permiten una diseminación más efectiva del virus, de forma que incluso los viriones infectantes logran alcanzar el estrato espinoso, sitio donde como ya se mencionó, tiene lugar la integración del genoma viral. En este paso, se pierde la inhibición sobre región promotora inducida por el dímero E1-E2, que impedía la síntesis de las proteínas E6 y E7. A causa de la pérdida del efecto protector del p53 por activación de la proteína E6, se da inicio a una mayor amplificación del ADN viral mutado, con la consecuente proliferación de células infectadas, que finalmente culminará con la generación de lesiones de características neoplásicas ${ }^{13}$.

Como hemos observado, el ciclo de vida del virus se desarrolla en el epitelio genital, no requiere que se produzca viremia, y en general los bajos niveles de proliferación y la ausencia de destrucción celular masiva, impiden en gran medida la activación de procesos inflamatorios, además tampoco se da una extensa liberación de citoquinas que induzca la migración de células presentadoras de antígenos (APCs). Finalmente, otro de los mecanismos que utiliza el virus para evadir la activación del sistema inmunológico del hospedero, consiste en la supresión de IFNa e IFN $\beta$, citoquinas con actividad antiangiogénica, antiviral y antiproliferativa ${ }^{14}$.

A pesar de todos los esfuerzos virales por impedir la activación inmunológica, ninguno de los procesos descritos es $100 \%$ efectivo, por ejemplo, se sabe que aunque no hay viremia y que la infección está limitada al epitelio genital, pocas cantidades de partículas virales libres escapan del epitelio hacia canales linfáticos, desde los cuales se dirigen hasta los ganglios linfáticos, donde se inicia una respuesta celular inmunomediada ${ }^{14}$. Las principales partículas implicadas en dicha respuesta son las proteínas capsulares L1, capaces de inducir bajos niveles de anticuerpos inca- 
paces de generar seroconversión en la mayoría de humanos, pero con el potencial de aclarar cerca del $80-90 \%$ del virus infectante en las células hospederas ${ }^{15}$.

Respuesta inmunológica desarrollada a partir de la vacunación contra VPH

La debilidad en la respuesta inmune generada posterior a la infección natural por el VPH, llevó al desarrollo de estudios que trataron de explicar en modelos animales las diferencias existentes entre la infección natural y la generada, producto de la exposición a los antígenos de la cápside viral, ya fuera a través de la aplicación directa de los componentes virales de manera intramuscular o intravenosa o más recientemente a través de la aplicación de una vacuna creada por ingeniería genética a partir de partículas similares al virus (VLPs) ${ }^{15}$. Los VLPs son partículas no infectantes derivadas de las proteína L1 de la cápside viral expresadas en células de levadura ${ }^{12}$.

Dentro los estudios fase 3 más importantes que comprueban la efectividad de la vacuna derivada de VLPs, destacan las revisiones FUTURE I y II que lograron demostrar un nivel de protección del $98 \%$ contra lesiones de alto grado, así como del $100 \%$ contra lesiones vulvares y cervicales con el uso de la vacuna tetravalente, mientras que el estudio PATRICIA, logró demostrar una protección del $100 \%$ contra lesiones de alto grado con la vacuna bivalente. Así mismo, se ha confirmado que al aplicar cualquiera de las vacunas en esquemas de tres dosis, se desarrollan anticuerpos altamente eficaces y a títulos altos, que incluso generan protección por periodos de hasta 5,5 años ${ }^{8,16}$. Además, en prácticamente la totalidad de los participantes, se detectó seroconversión de los anticuerpos anti VLP, dominando el subtipo $\operatorname{IgG1}$, hallazgo escasamente detectado en la infección natural ${ }^{14}$.

La respuesta inmune generada tras la administración de la vacuna contra VPH inicia con la aplicación intramuscular de los VPH L1 VLPs, una vez en el tejido muscular esas partículas son rápidamente reconocidas por las APCs estroma- les y las células de Langerhans musculares, para posteriormente a través de los canales linfáticos acceder a los ganglios linfáticos regionales, sitio donde tiene lugar la activación de las células B y su posterior diferenciación hacia células plasmáticas o bien hacia anticuerpos. Esta forma de administración introduce el primer cambio y de los más importantes en el ciclo de vida del VPH, el cual de forma natural se limita a la infección intraepitelial ${ }^{17}$.

Al ser los VLPs moléculas altamente inmunogénicas tienen la capacidad de activar el sistema inmune innato y adaptativo, en ratones por ejemplo se ha demostrado que logran unirse a células dendríticas mieloides, a través de la señal mediada por receptores de células T (TCR) dependientes de la vía MYD88, la cual es esencial para la activación de las células B y la generación de anticuerpos. En humanos se supone que existe una vía similar, en la cual la generación de anticuerpos, depende de la activación de las células T. La respuesta $\mathrm{T}$ es iniciada por la APCs que presenta un epitopo, en este caso derivado del VLP, a través del complejo mayor de histocompatibilidad tipo II (MHC II), que permite la diferenciación hacia $\mathrm{Th} 2$, las cuales liberan entre otras la IL-4 e IL-10, que culminaran con la activación de las células $\mathrm{B}^{17}$.

La efectividad con la que se activa la respuesta $\mathrm{T}$ es variable, por ejemplo va a depender del haplotipo específico de MHC que cada individuo posee, de la cantidad y vía de administración del inmunógeno, y además dependerá de las moléculas coestimuladoras y citoquinas proinflamatorias que estén implicadas en la generación de la respuesta T. En relación a este último punto es que se ha trabajado en la producción de vacunas con adyuvantes, en su mayoría derivados de sales de Aluminio ${ }^{18}$. Los adyuvantes son moléculas capaces de aumentar o modular la inmunogenicidad intrínseca del antígeno. La respuesta T consta de tres señales, la primera dada por la activación del receptor de la célula T (TCR), la segunda deriva de las moléculas coestimuladoras y la tercera señal viene dada por las citoquinas proinflamatorias liberadas por las APCs, de forma natural en la infección por VPH la tercera señal 
deriva de los receptores tipo Toll (TLRs), mientras que en sujetos vacunados esta señal es generada por los adyuvantes agregados a la vacuna. Entonces, parte de lo que también explica la mayor efectividad de la respuesta inmune generada por la vacuna versus la producida por la infección natural, viene dada por la adición de adyuvantes a las formulaciones en que se comercializan las vacunas ${ }^{17}$.

Finalmente, otra de las ventajas asociadas a la vacunación con VLPs corresponde a la generalización no sólo de anticuerpos específicos contra alguno de los serotipos incluidos en la vacuna (6, 11,16 y 18), ya que tras la administración de los VLPs se ha demostrado la existencia de protección cruzada contra otros serotipos, aunque no se encuentren incluidos en la vacuna. En relación a este fenómeno se ha logrado determinar que los VLPs al ser moléculas tan complejas, contienen múltiples epitopos y demás determinantes antigénicos, que activan diferentes respuestas $\mathrm{T}$ dependientes que culminan con la producción de diferentes anticuerpos, algunos de ellos inmunodominantes o tipo específicos, y otros que corresponden a subpoblaciones de anticuerpos compartidos por otros serotipos de $\mathrm{VPH}^{15}$.

Introducción de esquemas de vacunación contra VPH basados en dos dosis

Habiéndose demostrado la eficacia inmunológica de los esquemas de tres dosis, en relación a la generación de anticuerpos protectores contra enfermedades derivadas de la infección por $\mathrm{VPH}$, es que han surgido múltiples revisiones donde se ha logrado demostrar que existe eficacia comparativa en lo referente a seroconversión entre un esquema de dos dosis y el esquema tradicional de tres dosis. Estos esfuerzos han sido guiados principalmente, en el contexto de que un esquema de dos dosis resulta más accesible en cuanto a costos y apego a tratamiento por parte de la población meta. Por tanto, un esquema de dos dosis podría conducir a un aumento considerable del número de individuos que completen el esquema, con lo cual se asegura un mayor porcentaje de protección ${ }^{11}$.
Dentro de los estudios iniciales que evaluaron estos esquemas de dosificación se encuentra el análisis de Romanowski y colegas, estudio controlado, aleatorizado que compara los esquemas de tres dosis versus dos dosis en cuanto a inmunogenicidad de la vacuna bivalente contra el VPH-16 y 18. En éste, se agruparon las mujeres por edades: 9 a 14, 15 a 19 y 20 a 25 años y se concluye que no existe diferencia entre los esquemas valorados al mes y a los 24 meses post última dosis aplicada. Por otro lado, se observó que el esquema de 3 dosis aplicado al grupo de 9-14 años presentó niveles de anticuerpos mayores en comparación a dos dosis, sin saberse las implicaciones a largo plazo; además el esquema de dos dosis acortado, 0 y 2 meses evidenció disminución en el título de anticuerpos, de lo que se puede rescatar que se requiere un intervalo de tiempo considerable entre una primera dosis y la segunda para lograr una adecuada respuesta inmunológica ${ }^{19}$.

Posteriormente en Costa Rica, el análisis llevado a cabo por Safaeian y colaboradores, analiza la persistencia de los anticuerpos entre los grupos de pacientes que recibieron una sola dosis, dos dosis separadas por un mes, dos dosis separadas por seis meses, y tres dosis. Se determinó la magnitud y la durabilidad de los anticuerpos contra HPV-16 y 18 mediante visitas anuales a través de cuatro años, donde se observó que el $100 \%$ de las mujeres en todos los grupos se mantuvieron seropositivos para HPV-16 y 18. Además, no se demostró inferioridad entre el grupo que recibió dos dosis separadas por seis meses y el que recibió tres dosis. Se determinó dentro de los hallazgos que lo títulos de anticuerpos contra HPV 16 y 18 se encontraron al menos, 24 y 14 veces mayor entre los grupos que recibieron dos dosis y 9 y 5 veces más altos entre los vacunados con una sola dosis, comparando ambos grupos con los niveles de los títulos obtenidos por infección natural. Otro aspecto valorado fue que los títulos se mantuvieron estables desde el mes 6 al 48. Por tanto se podría plantear que incluso una sola dosis podría inducir protección a largo plazo, sin embargo se requieren estudios que mantengan el seguimiento a través de los años, para 
valorar el impacto clínico de la protección inducida por la vacunación según los niveles de anticuerpos generados con cada esquema ${ }^{20}$.

Por otra parte, Kreimer y colaboradores, evaluaron la eficacia de la vacuna bivalente después de cuatro años de seguimiento, en mujeres jóvenes (15-25 años) mediante la suma de los datos suministrados por dos ensayos clínicos (CVT y PATRICIA), ambos fase 3, controlados, aleatorizados y doble ciego. Dentro de la población analizada 22327 mujeres recibieron tres dosis, 1185 dos dosis, 543 una dosis y se mantuvo una eficacia contra la incidencia de la infección para VPH-16 y 18 para el esquema de tres dosis fue de $77 \%$, para dos dosis $76 \%$ y para una dosis fue de $85.7 \%$. Además se comparó la eficacia de la vacuna contra VPH-16 y 18 para quienes recibieron dos dosis separadas por un intervalo de un mes y los que la recibieron 6 meses posterior a la primera dosis, para lo cual se obtuvo fue de $75.3 \%$ y de $82.6 \%$ respectivamente. La eficacia contra HPV-31, 33 y 45 para las mujeres que recibieron dos dosis separadas por seis meses fue de $68.1 \%$, similar a la obtenida por el grupo que recibió sus tres dosis, pero fue muy baja para los que recibieron su segunda dosis al mes (10\%). De lo que podemos concluir que 4 años posterior a la vacunación de mujeres de 15-25 años, los esquemas de una y dos dosis de la vacuna contra el VPH-16 y 18 parecen proteger contra las infecciones del VPH-16 y 18, con una eficacia similar a la suministrada por el esquema de tres dosis. Además que dos dosis separadas por 6 meses, brindan algún porcentaje de protección cruzada. Estos resultados sugieren que al aplicarse un esquema de dos dosis separados por seis meses se consigue una eficacia comparable a la obtenida con los esquemas de tres dosis ${ }^{21}$. El análisis de una sola dosis debe hacerse con precaución, pues es difícil esperar que una sola dosis active el sistema inmune de forma tal que se presente una memoria a largo plazo, según lo analizado en lo que respecta a la activación del sistema inmune.

Dobson y colegas, realizan un estudio multicéntrico, fase 3 , en el cual se utilizó la vacuna tetra- valente contra el VPH, en niñas de 9 a 13 años de edad que recibieron 2 dosis de la vacuna ( 0 y 6 meses) y mujeres jóvenes de 16 a 26 años de edad que recibieron 3 dosis de la vacuna $(0,2$ y 6 meses), cuyo punto final primario consistía en el análisis del título geométrico medio (GMT) para VPH-16 y 18, un mes posterior a la última dosis de la vacuna y se concluye que no hay inferioridad del esquema de dos dosis versus el de tres dosis para el punto primario evaluado. Sin embargo, dentro del análisis de los puntos secundarios se concluyó que la respuesta de los anticuerpos para VPH-18 al mes 24 y VPH-6 al mes 36 fue mayor para las niñas que recibieron 3 dosis versus las niñas que recibieron 2 dosis, de lo cual se deduce que siendo esta parte de la población meta de la vacunación, se requieren más estudios para evaluar las implicaciones clínicas a largo plazo de estos hallazgos ${ }^{22}$.

Los estudios que han analizado la aplicación de dos dosis de la vacuna contra VPH deben considerar dentro de sus resultados la implementación de modelos matemáticos, a través de los que se logre determinar la diferencia respecto a la protección inducida por los anticuerpos contra VPH, a través del tiempo. De modo que al utilizar dichos modelos incluso se puede concluir que los años de protección brindado por el esquema de dos dosis son determinantes para valorar la necesidad y el beneficio de una tercera dosis en particular. Por ejemplo, en el estudio de Jit y colaboradores, los modelos matemáticos utilizados sugieren que si el esquema de dos dosis protege por al menos 20 años, los beneficios de una tercera dosis es pequeño, por otro lado si dos dosis protegen por 10 años el número de casos de cáncer que se pueden prevenir con una tercera dosis aumenta ${ }^{23}$.

En la Unión Europea la vacuna bivalente contra el VPH con un régimen de dos dosis ha recibido el visto bueno para su comercialización, por otro lado la vacuna cuadrivalente ha recibido opiniones positivas de parte de la Agencia Europea de Medicamentos. Los esquemas de dos dosis se han adoptado en: Quebec, Suiza, Holanda y México ${ }^{23}$. 
A pesar de los hallazgos demostrados a través de los estudios mencionados con anterioridad, no basta con el análisis de sólo esas variables para decidir sobre la implementación de uno u otro esquema de vacunación, ya que deben valorarse otros contextos, por ejemplo aquellas personas con algún tipo de inmunodeficiencia o algún grado de desnutrición que pueda comprometer la respuesta inmune ante las vacunas ${ }^{24}$. Se deben de valorar también las diferencias entre las presentaciones disponibles por ejemplo Cervarix cuenta con dos adyuvantes potentes en cuanto a la activación de las células $\mathrm{B}$, una sal de aluminio simple y un agonista del receptor de tipo toll (TLR); otras presentaciones no cuentan con el receptor agonista tipo toll, por lo que no se pueden traslapar los resultados obtenidos en los estudios en los que se utilice una $u$ otra presentación en cuanto al efecto de reducción de dosis ${ }^{20}$. En estudios posteriores, también se requerirá del análisis en cuanto al impacto clínico de las nuevas presentaciones de la vacuna Gardasil $9^{\circledR 24}$.

Respuesta inmune inducida con esquemas de dos de la vacuna contra VPH

Se bien, existen varias revisiones que indican que los esquemas de 2 y 3 dosis pueden compararse en cuanto a la respuesta inmune, se requiere de más investigación para valorar el impacto a largo plazo en esta respuesta y el grado de protección cruzada que se brinda con las vacunas con respecto a los serotipos del VPH. Al ser la población meta personas jóvenes, con vida sexual activa, con largas expectativas de vida, se hace necesario valorar la protección generada con la vacunación en cantidad de años y de esta forma determinar si se requerirá de algún tipo de refuerzo a largo plazo ${ }^{24}$.

Para el análisis de los determinantes que pueden influir en la activación y la memoria de la respuesta inmune, como la edad y el número de dosis, estudios iniciales en ratones demostraron que una sola dosis de la vacuna contra el VPH 16 fue capaz de inducir niveles elevados de IFNy, sin embargo, tres dosis los elevaron incluso hasta seis veces más. Respecto a los niveles de IL-4 se detectaron solamente en los que se aplicaron tres dosis ${ }^{25}$. En relación a dichos hallazgos, vale la pena rescatar que en este momento se utilizó un preparado sin adyuvantes y que los modelos en este estudio fueron ratones.

Otro determinante que influye en el desarrollo de una respuesta inmune efectiva tras la aplicación de tan sólo dos dosis de la vacuna corresponde al tiempo que transcurre tras la primera y la segunda aplicación, ya que la respuesta inmune de las células B de memoria provocada por la primera dosis requiere al menos 4-6 meses para madurar y diferenciarse ${ }^{26}$. Se puede deducir que al considerar la aplicación de este nuevo esquema se requiere un mínimo de separación entre las dosis para conseguir la respuesta inmune deseada y el beneficio a largo plazo.

Por otra parte, en el estudio de Smolen y colaboradores en el cual se comparó la respuesta de células B en los grupos etarios de 9-13 años versus 16-26 años tras recibir un esquema de tres dosis, se concluyó que la edad influye en los niveles de células $\mathrm{B}$ de memoria específicas contra VPH-18, pues en el grupo de 16-26 años el nivel de células $\mathrm{B}$ específicas activadas con VPH-18 fue más bajo. Por otro lado, los niveles de IFN-y (producidos por las células T) fueron significativamente más bajos en las niñas que recibieron dos dosis cuando se compararon con las niñas y mujeres que recibieron tres dosis ${ }^{27}$. De esto, se puede concluir que tanto el número de dosis como la edad de aplicación pueden influir en la magnitud de la respuesta inmune, sin embargo no se cuenta con suficiente información para medir el impacto clínico a largo plazo.

Efecto inducido en la incidencia de cáncer cervical en mujeres vacunadas con esquemas de dos dosis contra VPH

El efecto de la vacunación se ha evaluado en algunos estudios clínicos, sin embargo, la medición propiamente en la incidencia del cáncer es difícil de estimar de manera confiable en la mayoría de los ensayos clínicos, pues el tiempo de seguimiento es relativamente corto y la aparición 
de neoplasias en la mayoría de estudios es baja. Por otro lado, si se ha documentado el efecto del esquema de dos dosis en la incidencia de infección por VPH y la presencia de lesiones premalignas de cérvix, así como también el impacto en incidencia de verrugas genitales, por lo que la evidencia presentada se enfoca en esos puntos clínicos. Además, la mayoría de las revisiones que evalúan el efecto de esquemas reducidos son estudios observacionales de casos y controles después de la introducción de los programas nacionales de vacunación de VPH en países desarrollados ${ }^{10}$.

De forma general, retomando la comparación entre eficacia de los diferentes esquemas, tenemos que en Escocia se documentó una disminución importante en la infección por serotipos 16 y 18 del virus al recibir dos o tres dosis de la vacuna bivalente; sin embargo solo la aplicación de 3 dosis mostró protección cruzada significativa; es decir una reducción significativa en la incidencia de infección de serotipos oncogénicos no vacunales ${ }^{28}$. Otra publicación demostró una disminución del riesgo de infección por VPH conforme aumenta el numero de dosis de vacuna tetravalente; sin embargo los pacientes que recibieron menos que dos dosis incluidas en el estudio fueron de una edad mayor a los que recibieron esquema completo, por lo que podrían ya haber estado expuestas a infeccion natural por el virus, lo que podría disminuir la eficacia de la vacuna $^{29}$.

En cuanto al efecto propiamente de lesiones premalignas $\mathrm{y}$ malignas de cervix, se ha evidenciado en Australia que en mujeres inmunizadas con vacuna tetravalente presenta una disminución importante en el riego de desarrollo de lesiones intraepiteliales de bajo grado para los esquemas de dos o tres dosis al comparar con las mujeres no vacunadas. Sin embargo, para la prevención de lesiones intraepitelales de alto grado, a pesar de que todos los grupos vacunados presentaron una disminución en su incidencia así como también para adenocarcinoma, solo mostraron una disminución significativa en el riesgo las pacientes que recibieron un esquema completo de 3 dosis; lo que indica que los esquemas con menos de tres dosis podrían no conferir protección contra lesiones de alto grado; sin embargo se deben realizar más estudios; puesto que las pacientes que recibieron una menor cantidad de dosis eran de mayor edad y de clases socioeconómica baja, lo que podría indicar un riesgo alto de haber estado infectadas por VPH antes de la vacunación ${ }^{30}$.

En un estudio realizado en Dinamarca, se evaluó el efecto de la vacuna tetravalente en el riesgo de aparición de verrugas genitales. En esta publicacion, a pesar de que no se estratifica el riesgo por el número de dosis recibidas; se comprueba que se da una disminución significativa en el riesgo de verrugas genitales en las niñas que recibieron al menos una dosis al compararlas con la niñas no vacunadas (riesgo varía desde 0.12 a 0.62 dependiendo de la edad) presentando un menor riesgo en pacientes más jóvenes ${ }^{31}$. En Suecia, una cohorte abierta de mujeres entre los 10-24 años, evaluó la incidencia relativa y la diferencia de incidencias relacionado con la cantidad de dosis recibidas de vacuna tetravalente, estratificado de acuerdo a la edad en la que se recibió la primera dosis; 10-16 años o de 17-19 años. Dentro de los resultados, se obtuvo una disminución en el riesgo de incidencia en el estrato de 10 a 16 años, dependiente del número de dosis: 0.18, 0.29 y 0.31 (tres, dos o una dosis; respectivamente). Mientras que en las pacientes que recibieron la primera vacuna entre los 17-19 años se presentó una tasa de incidencia para los que recibieron tres, dos o una dosis de vacuna tetravalente de $0.23,0.35$ y 0.71 ; respectivamente. Estos hallazgos no son del todo inesperados; pues según lo expuesto anteriormente se presenta una mayor inmunogenicidad en las pacientes más jóvenes. Además, se estima que el número de casos de condilomas prevenidos con 3 dosis en comparación con 2 dosis de la vacuna son de 56 casos por cada 100000 años persona, lo cual no representa una gran diferencia ${ }^{32}$. Por otra parte no se conoce si esta tendencia se relaciona directamente con enfermedad neoplásica. 
No obstante, como algunas de estas publicaciones sugieren una adecuada efectividad del esquema de dos dosis, la WHO ha llegado a cambiar la recomendaciones respecto a la vacunación contra VPH, indicando la vacuna para niñas entre los 9 y 13 años. Con aclaración de que las pacientes que recibieron la primera dosis antes de los 15 años pueden utilizar el esquema de dos dosis; siendo estas separadas por un periodo de al menos 6 meses. Si el intervalo es menor a 5 meses se recomienda una tercera dosis; también se indica que en pacientes inmunosuprimidas (infección por HIV o secundario a transplante de órganos), así como en pacientes mayores de 15 años deben recibir el esquema de tres dosis ${ }^{33}$.

Costo-efectividad de los esquemas de dos dosis contra VPH

Como consecuencia de la evidencia que sugiere que tras la administración de dos dosis de la vacuna tetra o bivalente contra VPH se obtiene una protección similar a la documentada con los esquemas de tres dosis, durante un periodo de alrededor de tres años, es que han surgido nuevos análisis a fin de comparar la costoefectividad de estos nuevos esquemas en relación a los anteriores, basados en tres dosificaciones ${ }^{34}$. En ciertos países como Canadá, Reino Unido, Estados Unidos y Australia la introducción de los esquemas de vacunación contra VPH se han popularizado, ocupando cerca del $40 \%$ del presupuesto destinado a la inversión en inmunizaciones para niños y jóvenes al menos en Canadá, de manera que la instauración de un nuevo esquema que reduzca el número dosificaciones, sin disminuir la protección contra enfermedades derivadas del VPH, permitiría a las naciones invertir en otros proyectos de inmunización e incluso facilitaría el acceso de la población masculina al esquema básico contra VPH, ya que en la actualidad en la mayoría de países los esquemas se enfocan en solo mujeres ${ }^{35}$.

Los estudios de costoefectividad en relación a estos nuevos esquemas, se han realizado en base a cuatro diferentes modelos comparativos que derivan de modelos matemáticos, que suponen la perpetuación de la protección a través de los años. El primero basado en la comparación entre los esquemas de dos dosis en mujeres versus la no aplicación de la vacuna, el segundo consiste en comparar tres dosis solo en mujeres versus dos dosis solo en mujeres, el tercer modelo compara los esquemas de dos dosis en mujeres y hombres versus solo en mujeres y el cuarto compara tres dosis en mujeres y hombres versus tres dosis solo en mujeres o dos dosis en mujeres y hombres. Se ha logrado determinar que la aplicación de los esquemas de dos dosis sólo en mujeres, cuando su duración perdure por lo menos durante 10 años, logran reducir en un $13 \%$ la probabilidad de cáncer asociado a la infección por VPH y entre un 54-60\% de las lesiones anogenitales, por otra parte al aplicarse esquemas de tres dosis solo en mujeres se consigue una reducción del 13-15\% adicional en la incidencia de cánceres asociado a VPH, mientras que si se aplican esquemas de dos dosis en hombres y mujeres se consigue una protección adicional del $3 \%$ en cánceres y del $9 \%$ en verrugas anogenitales. Sin embargo, si los esquemas de dos dosis en cualquiera de los ejemplos anteriores se extienden por los menos durante 20-30años, los beneficios de los esquemas de tres dosis se reducen, brindando una protección de solo un 2-6\% adicional en cuanto a la incidencia de cáncer ${ }^{35}$.

De tal modo, que para que los esquemas de dos dosis sean costoefectivos en relación a no aplicar la vacuna, se requiere que la protección generada por este esquema sea de por lo menos 10 años, mientras que para que los esquemas de dos dosificaciones sean costoefectivos, si se comparan con esquemas de tres dosis, se requiere de al menos una protección que perdure durante 30 años. Evento explicado en relación a que se estaría protegiendo de la infección durante el pico de mayor actividad sexual (de los 18-35años) y por ende se reduciría la probabilidad de infección en el grupo etario más vulnerable. Por otra parte al comparar la administración de esquemas de dos dosis en mujeres y hombres versus tres dosis solo en mujeres, para que el primer esquema sea costoefectivo se requiere que el costo por dosis 
en hombres sea sustancialmente menor en comparación con cada dosis aplicada en mujeres ${ }^{35}$.

Finalmente, se podría concluir que la efectividad tras la implementación de uno $\mathrm{u}$ otro esquema dependerá de la capacidad de dicho esquema para generar una protección duradera a través del tiempo. Por lo tanto, es en base a este hecho que se deberían fundamentar las decisiones respecto a cuál esquema implementar, sin embargo el cálculo de la duración generada por cada esquema, no es un dato fácil de precisar ni que en actualidad se conozca con certeza ${ }^{35}$.

\section{CONCLUSIONES}

Con relación a la introducción de nuevos esquemas simplificados de vacunación contra VPH, hasta el momento se tienen datos preliminares que demuestran seguridad al utilizar esquemas de dos dosis separadas por un lapso de al menos seis meses en mujeres jóvenes (15 años o menos), lo cual sin duda genera un impacto positivo a nivel de acceso a la vacunación y porcentaje de población cubierta.

Por otro lado, siguen existiendo interrogantes en lo referente a calidad de la protección cruzada, nivel óptimo de anticuerpos capaz de generar protección clínica, y tiempo de duración de la respuesta inducida por uno u otro esquena, que asegure su costoefectividad, por lo que de momento quedamos a la espera de más estudios clínicos que traten de esclarecer estos puntos.

\section{BIBLIOGRAFÍA}

1. Munro A, Cruickshank M. Impact of HPV immunization on the detection of cervical disease. Expert Rev. Vaccines 2014;13:533544.

2. Buchanan T, Graybill W, Young J. Morbidity and Mortality of Vulvar and Vaginal Cancers: Impact of 2, 4-, and 9valent HPV Vaccines. Human Vaccines \&
Immunotherapeutics 2016; Feb 22:0. [Publicación para impresión].

3. Barr E, Sings H. Prophylactic HPV vaccines: New interventions for cancer control. Vaccine 2008;26: 6244-6257.

4. Palefsky J, Giuliano A, Goldstone S, et al. HPV Vaccine against Anal HPV Infection and Anal Intraepithelial Neoplasia. N Engl J Med 2011;365: 1576-1585.

5. Palefsky, J. Can HPV vaccination help to prevent anal cancer? The Lancet 2010;10:815-816.

6. Luckett R, Feldman S. Impact of 2-, 4- and 9- valent $H P V$ vaccines on morbidity and mortality from cervical cancer. Human Vaccines \& Immunotherapeutics 2015; Nov 20:0. [Publicación para impresión].

7. Committee on Adolescent Health Care Inmunization Expert Work Group. Human Papillomavirus Vaccination. ACOG 2015;641:1-6.

8. Bayas J, Costas L, Muñoz A, Cervical cancer vaccination indications, efficacy, and side effects. Ginecologic Oncology 2008;110:11-14.

9. Giuliano A, Palefsky J, Goldstone S. Efficacy of Quadrivalent HPV Vaccine against HPV Infection and Disease in Males. N Engl J Med 2011;364:401-411.

10. Toh Z, Licciardi P, Fong J, et al. Reduced dose human papillomavirus vaccination: An update of the current state-of-the-art. Vaccine 2015;33:5042-50.

11. Kreimer AR, Rodriguez AC, Hildesheim A, et al. Proof of principle evaluation of the efficacy of fewer than three doses of a bivalent HPV16/18 vaccine. J Natl Cancer Inst 2011;10:1444-51.

12. Villa L, Ault $\mathrm{K}$, Giuliano $\mathrm{A}$, et al. Immunologic responses following administration of a vaccine targeting human papillomavirus Types 6, 11, 16, and 18. Vaccine 2006;24:5571-5583. 
13. Rivera R, Delgado J, Painel V, et al. Mecanismo de infección y transformación neoplásica producido por virus papiloma humano en el epitelio cervical. Rev Chil Obstet Ginecol 2006;71:135-140.

14. Stanley M. Immune responses to human papillomavirus. Vaccine 2006;24S1:16-22.

15. Stanley M. Immunobiology of HPV and $H P V$ vaccines. Gynecologic Oncology 2008;109:15-21.

16. Gattoc L, Nair N, Ault K. Human Papillomavirus Vaccination: Current Indications and Future Directions. Obstet Gynecol Clin N Am 2013;40:177-197.

17. Stanley M, Gitssmann L, Nardelli-Haefliger D. Immunobiology of Human Papillomavirus Infection and VaccinationImplications for Second Generation Vaccines. Vaccine 2008;26:62-67.

18. Ault K. Long-term efficacy of human papillomavirus vaccination. Gynecologic Oncology 2007;107:27-30.

19. Romanowski B, Schwarz TF, Ferguson LM, et al. Immunogenicity and safety of the HPV-16/18 AS04-adjuvanted vaccine administered as a 2-dose schedule compared with the licensed 3-dose schedule: results from a randomized study. Hum Vaccin. 2011;7:1374-1386.

20. Safaeian M., Porras C., Yuanji Pan, et al. Durable Antibody Responses Following One Dose of theBivalent Human Papillomavirus L1 Virus-Like ParticleVaccine in the Costa Rica Vaccine Trial. Cancer Prev Res 2013;6:1242-1250.

21. Kreimer A., Struy F., Raymundo, M. et al. Efficacy of fewer than three doses of an HPV-16/18 AS04-adjuvanted vaccine: combined analysis of data from the Costa Rica Vaccine and PATRICIA trials. The Lancet Oncol 2015; 16: 775-86.

22. Dobson SR, McNeil S, Dionne M, et al. Immunogenicity of 2 doses of HPV vaccine in younger adolescents vs 3 doses in young women: a randomized clinical trial. JAMA. 2013;309:1793-1802.

23. Jit M, Choi YH, Laprise JF, et al. Two-dose strategies for human papillomavirus vaccination: How well do they need to protect?. Vaccine 2014;32: 3237-3242.

24. Kahn J, Bernstein D. HPV Vaccination Too Soon for 2 Doses?. JAMA 2013;309:18321833.

25. Marais D, Passmore JA, Maclean J, et al. A recombinant human papillomavirus (HPV) type 16 L1-vaccinia virus murine challenge model demonstrates cell-mediated immunity against HPV virus-like particles. J GenVirol 1999;80:2471-5.

26. Siegrist CA. Vaccine immunology. In: Plotkin WAO SA, Offit PA, editors. Vaccines. 5th ed. New York, USA: Elsevier; 2008. p. 17-36.

27. Smolen K, Gelinas L, Franzen L, et al. Age of recipient and number of doses differentially impact human $B$ and $T$ cell immune memory responses to $\mathrm{HPV}$ vaccination. Vaccine 2012;30:3572-3579.

28. Kavanagh K, Pollock KG, Potts A, et al. Introductionand sustained high coverage of the HPV bivalent vaccine leads to a reduction in prevalence of HPV 16/18 and closely related HPV types. $\mathrm{Br} \mathrm{J}$ Cancer 2014;110:2804-11.

29. Crowe E, Pandeya N, Brotherton JM, et al. Effectiveness of quadrivalent human papillomavirus vaccine for the prevention of cervical abnormalities: case-control study nested within a population based screening programme in Australia. BMJ 2014;348:1458.

30. Gertig DM, Brotherton JM, Budd AC, Drennan K, Chappell G, Saville AM. Impact of a population-based HPV vaccination program on cervical abnormalities: adata linkage study. BMC Med 2013;11:227.

31. Blomberg M, Dehlendorff C, Munk C, Kjaer SK. Strongly decreased risk of genital warts 
after vaccination against human papillomavirus: nation wide follow-up of vaccinated and unvaccinated girls in Denmark. Clin Infect Dis 2013;57: 929-34.

32. Herweijer E, Leval A, Ploner A, et al. Association of varying number of doses of quadrivalent human papillomavirus vaccine with incidence of condyloma. JAMA 2014;311:597-603.

33. World Health Organization (WHO). Human papillomavirus vaccines: WHO position paper, October 2014. Wkly Epidemiol Rec 2014; 89:465-92 (http://www.who.int/wer/2014/wer8943.pdf)

34. Aljunid S, Maimaiti N, Nur1 A, et al. Costeffectiveness of HPV vaccination regime: comparing twice versus thrice vaccinations dose regime among adolescent girls in Malaysia. BMC Public Health 2016;16:1-5.

35. Laprise JF, Drolet M, Boiy MC, et al. Comparing the cost-effectiveness of two and three dose schedules of human papilloma virus vaccination: A transmission-dynamic modelling study. Vaccine 2014;32:58455853.

\section{CONFLICTO DE INTERÉS Y/O AGRADE- CIMIENTOS}

Los autores declaran que no existió ningún conflicto de interés en el presente reporte. 